\title{
Therapeutic Implications of Chemically- and Biologically-Altered Sebum Being One Cause of Seborrheic Dermatitis
}

\author{
Craig G. Burkhart ${ }^{*}, 1$ and Craig N. Burkhart ${ }^{2}$ \\ ${ }^{I}$ University of Toledo School of Medicine, Ohio University College of Osteopathic Medicine, 5600 Monroe Street, Suite \\ 106B, Sylvania, Ohio 43560, USA \\ ${ }^{2}$ Department of Dermatology, Northwestern University, Chicago, Illinois, USA
}

\begin{abstract}
Background: Seborrheic dermatitis affects approximately 2 to 5\% of the populace. Interestingly, the term 'seborrheic dermatitis' is somewhat a misnomer as there is no direct quantitative relationship between the degree of sebaceous activity and susceptibility to seborrheic dermatitis.

Method: A pilot study was performed to initiate assessment of patient satisfaction with oral minocycline and topical ketoconazole for seborrheic dermatitis, and in so doing, set a stage for discussion for a novel assessment on the pathophysiology of this condition.

Results: Clinical improvement of seborrheic dermatitis occurred with oral minocycline and topical ketoconazole. In terms of all parameters studied, the condition improved with therapy and all patients desired to continue with the treatment regimen.

Conclusions: One could speculate that the clinical benefit of seborrheic dermatitis with antibiotics is likely via its effect on the microbiology within the sebaceous unit as well as on the alterations which thereby occur to the composition of the sebaceous secretions as they pass through the pilosebaceous unit. After being secreted, human sebum can be altered by various factors including secretion of hyaluronidase, proteases, and lipases by $P$. acnes. A closer examination of the alteration in sebum content both in the seborrheic condition and in treatment therapies warrants further investigation.
\end{abstract}

\section{INTRODUCTION}

Seborrheic dermatitis is a common inflammatory scaling skin condition that affects areas of the skin with sebaceous glands, specifically the face, chest, and upper back. It is characterized by marginated, erythematous, pruritic papules and plaques covered with a greasy scale. It affects approximately $2-5 \%$ of the populace. The incidence of seborrheic dermatitis has a steady increase with age after puberty, with a peak between 18 and 40 , although the condition remains frequent into the seventh decade of life. It is more slightly more common in males.

Although the term 'seborrheic' and sebum overproduction have been associated with the condition, the sebum secretion rate of seborrheic dermatitis patients is not necessarily increased [1]. Maturation of the sebaceous glands is to be a permissive factor for the development of the condition, as there is no direct quantitative relationship between the degree of sebaceous activity and susceptibility to seborrheic dermatitis. Additionally, qualitative abnormalities of the composition of sebum also have not been demonstrated.

Unlike contact irritant dermatitis which is pathophysiologically defined by four interrelated components, namely skin barrier disruption, epidermal skin changes, cytokine release, and nerve ending changes [2], seborrheic dermatitis lacks a coherent definition. The many persisting sources of

*Address correspondence to this author at the Ohio University College of Osteopathic Medicine, 5600 Monroe Street, Suite 106B, Sylvania, Ohio 43560, USA; Tel: 419-885-3403; Fax: 419-885-3401;

E-mail: cgbakb@aol.com perplexity relating to many general aspects of seborrheic dermatitis relates to the lack of standardized diagnostic criteria, high variability of estimates of prevalence, and controversies on etiology and pathomechanisms [3]. A scrutiny of the world's literature leads one to believe that little is known absolutely indisputable concerning this commonplace disorder. In short, all theories of pathogenesis of seborrheic dermatitis as primarily a disorder of sebum and/or sebum excretion rate have proved fruitless. At least one author has suggested that the term 'seborrheic dermatitis' is a misnomer [3]. Fruitful research and clinical studies can only be achieved when other etiological tenets are proposed and studied.

To this end, a pilot study was performed to initiate assessment of patient satisfaction with oral antibiotics and topical ketoconazole for seborrheic dermatitis, and in so doing, set a stage for discussion for a novel assessment on the pathophysiology of this condition.

\section{STUDY DESIGN}

The study was an open-label comparison survey designed to examine patient satisfaction with oral minocycline $50 \mathrm{mg}$ twice daily and topical nizoral cream $v s$ their previous topical therapies. Fifteen patients, ranging in age from 18 to 62 and demonstrating chronic erythema covered with greasylooking scales on their scalp, face, mid-chest, and/or mid back were recruited from September 2006 through February 2007 to participate in the study. The patients selected had no history of atopy, connective tissue disease, or serious systemic ailments. None of the study people were pregnant, breast-feeding, nor on birth control pills or other antibiotics. 
Patients demonstrating any comedones, acne pustules, or acne papules were excluded.

All participants were given prescriptions for a two month quantity of oral minocycline and topical ketoconazole cream to be applied once daily to the affected skin area. After two months of treatment, patients completed a 5-question survey. Patients received no honorium for completing the study.

Participants were seen only at baseline and at the completion of the study. Patients who had previously used topical steroids were warned that there might be a flare of the erythema in the initial two weeks of therapy. Previous therapies of the 15 participants included nizoral cream (3), ciclopriox (1), topical steroids (5), and metronidazole (3). None of the study group had ever received oral antibiotics for their skin eruption.

The survey questions posed to the patients are presented in Table 1. Utilizing a 10-point scale ('treatment didn't help at all' to 'treatment did a fantastic job'), patients were asked to rate their response to several clinical parameters. The institutional review board of The Toledo Hospital was used.

\section{RESULTS}

Questionnaires were returned by all 15 patients, eight of whom were male. All 15 participants stated that the antibiotics with topical ketoconazole improved their condition and desired to remain on therapy.

The patients' ratings of this therapy in relation to past therapies on several clinical parameters were tabulated, and the averages shown in Table 2. The minocycline/ketoconazole cream therapy worked best to reduce the parameter of redness of seborrheic dermatitis, but scored almost equally as well in reducing the extent of skin involvement, and scaliness queries.

\section{CONCLUSIONS}

This pilot study revealed clinical improvement of seborrheic dermatitis with oral antibiotics and topical ketoconazole. All patients queried felt that their condition improved with therapy and all desired to continue with treatment. Of note, four patients had previously used topical ketoconazole and/or ciclopirox, but noticed better improvement with the addition of oral antibiotics. From this small sampling, the parameter of erythema associated with seborrheic dermatitis improved the most, with the extent of involvement and scaliness recorded slightly lower improvement scores.

Although many reasons could be speculated for the improvement of seborrheic dermatitis with oral antibiotics, for the development of this new hypothesis, it will be assumed that the benefit is via its effect on the microbiology within the sebaceous unit as well as on the alterations which thereby occur to the composition of the sebaceous secretions as they pass through the pilosebaceous unit. It will also be assumed that the anti-inflammatory activity of minocycline is due to its effects on the acne biofilm, as will be discussed below.

To begin, the microbiology of the pilosebaceous unit involves three coexisting groups of microorganisms: grampositive, coagulase-negative cocci (staphylococci and micrococci), anaerobic/aerobic diphtheroids (Propionibacterium acnes and Propionibacterium granulosum), and lipophilic yeasts (Pityrosporum species).

The staphylococci and micrococci are strict aerobes; therefore, their site of growth within the sebaceous unit is superficial, and these organisms are unable to reside in the anaerobic conditions of the infra-infundibulum where the major alterations in sebum occur.

The lipophilic yeasts that reside in hair follicles have been divided morphologically into Pityrosporum ovale and Pityrosporum orbiculare. These represent a complex of organisms and have been reclassified as Malassezia furfur as well as other Malassezia species. These organisms are regularly isolated from lesions of seborrheic dermatitis, and a few authors have attempted to relate the condition to infection with Pityrosporum ovale. However, there is no simple stoichiometric relationship between yeast number and severity of seborrheic dermatitis; unaffected skin may carry a load of organisms similar to lesions of seborrheic dermatitis [4]. As a result, the proportion of effectively treated patients with ketoconazole cream with seborrheic dermatitis is only $25.3 \%$, in which the vehicle gel scored $13.9 \%$ success [5]. In short, Malassezia would not seem to play a significant etiologic role in pathogenesis, and is probably a secondary event [6]. Moreover, the effect of ketoconazole topically for seborrheic dermatitis may be related to ketoconazole's effects on

Table 1. Survey Questions Posed to Seborrheic Dermatitis Patients After Two Months of Therapy with Oral Minocycline and Topical Ketoconazole

\footnotetext{
1. Did the use of minocycline and topical ketoconazole cream improve your condition?

Yes No

2. Do you wish to continue using minocycline and topical ketoconazole cream for your condition?

Yes No

For the next set of questions, grade on a scale of 1-10, with 1 meaning the treatment didn't help at all, and 10 meaning that the treatment worked fantastically, after two months of therapy.

3. How well did the treatment using minocycline and topical ketoconazole cream reduce the extent of the rash of seborrheic dermatitis?

$\begin{array}{lllllllll}1 & 2 & 3 & 4 & 5 & 7 & 8 & 9 & 10\end{array}$

4. How well did the treatment using minocycline and topical ketoconazole cream reduce the redness associated with the rash of seborrheic dermatitis?

$\begin{array}{lllllllll}1 & 2 & 3 & 4 & 5 & 7 & 8 & 9 & 10\end{array}$

5. How well did the treatment using minocycline and topical ketoconazole cream reduce the scaliness associated with the rash of seborrheic dermatitis?

$\begin{array}{lllllllll}1 & 2 & 3 & 4 & 5 & 7 & 8 & 9 & 10\end{array}$
} 
lipids, lipoproteins, apolipoproteins, inhibition of cytochrome p450, suppression of glucocorticoid synthesis, decreasing androgenic effects, and increasing lanosterol [7-9].

Table 2. Averages from Patient Satisfaction of Clinical Parameters on the 10-Point Scale Used in the Survey

\begin{tabular}{|l|c|}
\hline Query & Average Score \\
\hline \hline $\begin{array}{l}\text { How well did the treatment using minocycline and } \\
\text { topical ketoconazole cream reduce the extent of the } \\
\text { rash of seborrheic dermatitis? }\end{array}$ & 7.5 \\
\hline $\begin{array}{l}\text { How well did the treatment using minocycline and } \\
\text { topical ketoconazole cream reduce the redness } \\
\text { associated with the rash of seborrheic dermatitis? }\end{array}$ & 8.2 \\
\hline $\begin{array}{l}\text { How well did the treatment using minocycline and } \\
\text { topical ketoconazole cream reduce the scaliness } \\
\text { associated with the rash of seborrheic dermatitis? }\end{array}$ & 7.4 \\
\hline
\end{tabular}

If the microbial flora is significant in the pathogenesis of seborrheic dermatitis, the most likely organism is Propionibacterium acnes. P. acnes and its immunopotentiating properties may be the direct instigator of the condition via its interaction with antibody and complement, its chemotactic properties and its effects on cell-mediated immunity. This organism has been shown serologically and biochemically to be identical to Corynebacterium parvum, a potent stimulator of the reticulocendothelial system $[10,11]$. The organism has been used as an immunostimulatory adjunct in chemotherapy of numerous tumors $[10,12]$. P. acnes is overwhelmingly the predominant microorganism in the normal pilosebaceous follicle and has been divided into two serotypes and five biotypes. Up to $10^{7}$ viable $P$. acnes have been isolated from a single sebaceous unit. P. acnes is not pathogenic by normal standards because there is no correlation between the number of bacteria and the severity of seborrheic dermatitis or acne. However, $P$. acnes secretes several extracellular products that may be significant in seborrheic dermatitis. These include hyaluronidase, proteases, lipases, and chemotactic factors for neutrophils, lymphocytes, and macrophages. The microenvironment of the pilosebaceous unit is likely to play a major role in the amount of exoenzymes that are produced by the organism because in vitro studies demonstrate that their production is altered by factors such as $\mathrm{pH}$ and oxygen tension.

Human intracellular sebum is composed of squalene, waxes, and triglycerides. Once secreted, the sebum is altered by various factors including $P$. acnes and the incorporation of enzymes including lipases from this organism as well as by contact with ambient oxygen. Oxygen and microorganisms transform 'native' sebum, such as lysis of triglycerides to fatty acids. Free fatty acids (which have a know antimicrobial effect) are formed from triglycerides by bacterial lipases, produced by the lipolytic $P$. acnes [13]. Certain components of this new complex mixture of molecules present in the sebum have been shown to be irritating and cytotoxic [14].

The biofilm concept is applicable to seborrheic dermatitis. A biofilm is a complex aggregation of microorganisms marked by the excretion of a protective and adhesive matrix. Within a biofilm, multiple microenvironments exist allowing for the same species of bacteria to live in diverse niches with varying rates of metabolism, replication, and responsiveness to antibiotic therapy. Microorganisms within biofilms are 50 to 500 times more resistant to antimicrobial therapies than free-floating (planktonic) bacteria. Bacteria in a biofilm have a natural antibiotic resistance not apparent on agar plates. Biofilms can be observed for function, adhesive properties, antibiotic resistance, protein expression, and existence of bacteriophages. Once a biofilm is established, there is a change in behavior by the bacteria that is triggered by many factors, including quorum sensing, as well as other mechanisms that vary between species. When a cell switches modes, it undergoes a phenotypic shift in behavior in which large suites of genes are up- and down-regulated. For example, incriminating studies such as API 20A systems, 16sRNA sequencing, DNA-DNA hybridization, XTT reduction method, and scanning electron microscopy have suggested that the $P$. acnes biofilm is the inciting factor for acne [15], orthopedic infections [16] and in fatal bacterial granulomas after trauma $[17,18]$.

The complete $P$. acnes genome supports the existence of the $P$. acnes biofilm. In short, the $P$. acnes genome contains clusters of genes involved in polysaccharide capsule biosynthesis of a glycocalyx polymer, which account for adherence to a surface in biofilm formation secreted by the organisms $[19,20]$. For example, there are several sequences granting the genomic machinery necessary to make biofilm, such as UDP-N-acetylglucosamine 2 epimerase and glycosyl transferases $[19,20]$. Indeed, these substances assisting adherence of the bacteria to the follicular wall are likely the incriminating cause of comedones as well [21].

The treatment of seborrheic dermatitis with minocycline is not directed at reduction in numbers of $P$. acnes per se, but for its effect on the $P$. acnes biofilm and the resultant sebum composition. We propose that the microbiological principle of biofilms might apply to seborrheic dermatitis in which alteration of the physical, biological, and chemical environment of the pilosebaceous unit may be the primary function of treatment for seborrheic dermatitis.

In short, therapy with minocycline can alter $P$. acnes biofilm activity, leading to alteration in sebum composition, which clinically improves seborrheic dermatitis. The manipulation of the sebum content via antibiotics and other methods certainly warrants further investigation.

\section{REFERENCES}

[1] Burton JL, Pye RJ. Seborrhea is not a feature of seborrheic dermatitis. BMJ 1982; 286: 1169-71.

[2] Burkhart CG. Contact irritant dermatitis and anti-pruritic agents: the need to address the itch. J Drugs Dermatol 2003; 2: 143-6.

[3] Mastrolonardo M, Diaferio A, Logroscino G. Seborrheic dermatitis, increased sebum excretion, and Parkinson's disease: a survey of (im)possible links. Medical Hypotheses 2003; 60: 907-11.

[4] Gupta AK, Kohli Y, Summerbell RC. Quantitative culture of Malassezia species from different body sites of individuals with or without dermatoses. Med Mycol 2001; 39: 243-51.

[5] Elewski B, Ling MR, Phillips TJ. Efficacy and safety of a new once-daily topical ketoconazole $2 \%$ get in the treatment of seborrheic dermatitis: a phase III trial. J Drugs Dermatol 2006; 5: 64650 .

[6] Wattanbe S, Kano R, Sato H, Nakamura Y, Hasegawa A. The effect of Malassezia yeasts on cytokine production by human keratinocytes. J Invest Dermatol 2001; 116: 769-72. 
[7] Cedeno J, Mendoza SG, Velazquez E, Nucete H, Spiers J, Glueck CJ. Effect of ketoconazole on plasma sex hormones, lipids, lipoproteins, and apolipoproteins in hyperandrogenic women. Metabolism: Clin Exp 1990; 39: 511-7.

[8] Casali AK, Hamdam JS. Effects of three azole derivatives on the lipids of different strains of Sporothrix schenckii. Canad J Micro 1997; 43: 1197-202.

[9] Humphreys WG, Obermeier MT, Chong S, et al. Oxidative activation of acylguanidine prodrugs: intestinal presystemic activation in rats limits absorption and can be inhibited by co-administration of ketoconazole. Xenobiotica 2003; 33: 93-106.

[10] Adlam C, Scott MT. Lymphoreticular stimulatory properties of Corynebacterium parvum and related bacteria. J Med Microbiol 1973; 6: 261-5.

[11] Burkhart CG, Burkhart CN, Lehmann PL. Acne: a review of immunologic and microbiologic factors. Postgraduate Med J 1999; 75: 328-331.

[12] Cantrell JL, Wheat RW. Antitumor activity and lymphoreticular stimulation properties of fractions isolated from Corynebacterium parvum. Cancer Res 1979; 39: 3554-5.

[13] Higaki S, Morohashi M. Propionibacterium acnes lipase in seborrheic dermatitis and other skin diseases. Drugs Under Exp Clin Res 2003; 29: 157-9.
[14] Saint-Leger D. Fonction sebacee normale et pathologique: des recherches au milieu du gue. Pathologie Biologie 2003; 51:275-8.

[15] Burkhart CN, Burkhart CG. Microbiology's principle of biofilms as a major factor in the pathogenesis of acne vulgaris. Int J Dermatol 2003; 42: 925-7.

[16] Ramage G, Tunney MM, Patrick S, Gorman SP, Nixon JR. Formating of Propionibacterium acnes biofilms on orthopedic biomaterial and their susceptibility to antimicrobials. Biomaterials 2003; 24: $3221-7$.

[17] Gao TW, Li CY, Zhao XD, Liu YF. Fatal bacteria granuloma after trauma: a new entity. Br J Dermatol 2002; 147: 985-93.

[18] Xu XL, Gao TW, Sun YQ. The study of pathogenic bacteria of fatal bacteria granuloma after trauma. Chin J Lab Med 2005; 28: 619-21.

[19] Bruggeman H, Henne A, Hoster F. The complete genome sequence of Propionibacterium acnes, a commensal of human skin. Science 2004; 305: 671-3.

[20] Burkhart CN, Burkhart CG. Genome sequence of Propionibacterium acnes reveals immunogenic and surface-associated genes confirming existence of the acne biofilm. Int J Dermatol 2006; 45: 872 .

[21] Burkhart CG, Burkhart CN. Expanding the microcomedone theory and acne therapeutics: Propionibacterium acnes biofilm produces glue that holds corneocytes together to form plug. J Am Acad Dermatol 2007; 57: 722-4. 